\title{
Immunohistochemical study of in vivo and in vitro IgA coating of candida species in vulvovaginal candidiasis
}

\author{
K Böhler, H Klade, Ch. Poitschek, A Reinthaller
}

\begin{abstract}
Objective-To evaluate whether quantitative or qualitative IgA deficiencies in cervicovaginal secretions can be identified in patients with recurrent vulvovaginal candidiasis.
\end{abstract}

Design-Prospective and controlled study.

Setting-Department of Dermatology, University of Vienna.

Subjects-30 patients with symptomatic and recurrent vulvovaginal candidiasis at the time of their presentation. 30 healthy women as a control group.

Intervention-Blood samples were drawn for measurement of serum IgA levels. Smears of the cervix and vagina were taken for direct microscopy and microbiological culture. Lavage of the vagina and ectocervix was performed with sterile saline solution for measurement of cervicovaginal IgA levels.

Main outcome measures-IgA levels of serum and cervicovaginal secretion evaluated by Single Radial Immunodiffusion. IgA labelling was demonstrated on fungal elements in vaginal smears and subcultured blastospores after incubation with vaginal secretions by immunohistochemistry.

Results-We could not find any significant difference of IgA levels in serum and cervicovaginal secretions between the symptomatic group and healthy controls ( $p$ value for serum $=0.5796$, $p$ value for secretion $=0.2381$ ). In vaginal smears yeasts revealed IgA coating on their surfaces, whereas three of the 61 subcultures were negative. Negative subcultures were assigned to three patients with recurrent candidiasis. No correlation was found between IgA levels of cervicovaginal secretions and staining intensity of subcultured blastospores after incubation with vaginal secretions $(r=-0.0578)$. IgA levels of serum and vaginal secretion showed no correlation $(r=-0 \cdot 00012)$.

Conclusion-Recurrent vulvovaginal candidiasis cannot be attributed to IgA deficiency. In some cases an IgA coating defect of yeasts might be involved. In addition inactivation of the IgA molecule by candida proteases might be of pathogenetic importance.

(Genitourin Med 1994;70:182-186)

Introduction

Candidal vaginitis is one of the most common causes of symptomatic vaginitis worldwide. Prevalence studies indicate that $20 \%$ of asymptomatic, healthy women of reproductive age carry Candida species in their vaginas. ${ }^{1}$ Several factors like pregnancy, the use of high oestrogen oral contraceptives and uncontrolled diabetes mellitus are associated with an increased asymptomatic vaginal colonisation as well as symptomatic disease. ${ }^{1}$ Clinical observations indicate that some women never develop symptomatic candidal vaginitis, some show infrequent, sporadic episodes of the disease and a third population suffers from recurrent, often chronic infection. ${ }^{23}$ Major controversy exists over the aetiology of recurrent vulvovaginal candidiasis (VVC). At present the three major causes, reinfection, non eradiction and overgrowth of candida, are suggested. ${ }^{4}$ The most likely infection routes of recurrent VVC are reinfection via the gastrointestinal tract and sexual transmission. However, attempts to reduce rectal candida carriage with nystatin caused a minor reduction of recurrence rates. ${ }^{5}$ No significant reduction in recurrence could be attained by concurrent treatment of sexual partners. ${ }^{4}$

Up to now it is not completely clarified why some women are more prone than others to recurrence of VVC. The role of cell mediated immunity in patients with recurrent VVC has been studied and a decreased lymphocyte proliferation has been found. ${ }^{6}$ Whitkin et al. suggest that prostaglandin secretion of macrophages might block the proliferative response of patients' lymphocytes. ${ }^{\text {? }}$

Although cellular immunity is important for the prevention of recurrent VVC it is unlikely to directly prevent overgrowth and adherence of candida species to vaginal epithelia. Adherence, the initial event in the development of overt disease, is strongly influenced by immunoglobulins, which are secreted onto mucous membrane surfaces. In cervicovaginal secretions secretory immunoglobulin A (sIgA) appears to be the most consistant and important constituent. ${ }^{8-10}$

The present experimental and immunohistochemical study was undertaken to identify any quantitative or qualitative deficiencies of sIgA in cervicovaginal secretions and to evaluate if low sIgA levels or sIgA coating defects of candida cells are detectable in patients with recurrent VVC.

Subjects and methods

Sixty non pregnant women aged between 17 and 50 years (mean 31.7 years) with regular menstrual cycles were included in the study 
after oral informed consent was obtained. Thirty women had a symptomatic candida vaginitis when they entered the study, demonstrating yeasts in their vaginal smears and/or cultures and a history of three or more episodes of VVC within the previous 12 months (group 1). Patients were investigated during the symptomatic episode irrespective of the phase of the menstrual cycle. Thirty consecutive, healthy women without previous VVC sustaining routine cervicovaginal investigation for other reasons were included as a control group (group 2). Vaginal smears and culture results of the controls were negative.

Gram stained smear preparations were analysed for epithelial cells, clue cells, leucocytes, hyphae and blastospores. High vaginal swabs for yeast culture were placed in liquid Sabouraud dextrose broth (containing 0.02 $\mathrm{mg} / \mathrm{ml}=0.02 \%$ bacitracin) and incubated at $37^{\circ} \mathrm{C}$ for 48 hours. Bacterial vaginosis, Trichomonas vaginalis, Neisseria gonorrhoeae, Chlamydia trachomatis, mycoplasma and ureaplasma-infection were excluded by culture.

Cervicovaginal secretions were collected from all 60 women. $10 \mathrm{ml}$ sterile $0.9 \%$ saline was instilled into the cervix and about $8 \mathrm{ml}$ of mucus and saline then aspirated from the vagina. The specimen were centrifuged at $1400 \mathrm{~g}$ for 15 minutes to deposit debris. The supernatants were concentrated by centrifugation at $55 \mathrm{~g}$ for 7 minutes in ultrafiltration cones (Centriprep 30 Concentrator 30000 molecular cut off, Amicon, Witten, Germany) and then lyophilised. Sampling and processing of cervicovaginal specimen was performed by one investigator to keep variations as minimal as possible. After reconstitution in $300 \mu \mathrm{l}$ phosphate-buffered saline (PBS) IgA levels were measured by single radial immunodiffusion (RID) (LC-Partigen IgA Immunodiffusionsplatte, Behring, Marburg, Germany, range $0.8-13.3 \mathrm{mg} / \mathrm{dl}$ ). The remaining $250 \mu 1$ were diluted to $2 \mathrm{ml}$ and stored at $-20^{\circ} \mathrm{C}$ for further immunohistochemical analysis.

Serum for IgA quantitation was available in 59 women. IgA levels were evaluated by single radial immunodiffusion (NOR-Partigen IgA Immunodiffusionsplatte, Behring; Marburg, Germany, range $54-1000 \mathrm{mg} / \mathrm{dl}$ ). Vaginal secretions were suspended in one drop of distilled water and dried on glass slides.

For obtaining subcultures of vaginal yeasts vaginal secretions were suspended in liquid Sabouraud dextrose broth containing 0.02 $\mathrm{mg} / \mathrm{ml}$ bacitracin and incubated at $37^{\circ} \mathrm{C}$ for 48 hours. Isolates were identified on the basis of API 20C Aux Candida (Bio Merieux). Yeast cultures of group 1 were subcultured twice on Sabouraud dextrose agar at $28^{\circ} \mathrm{C}$ for 48 hours. After washing steps, each followed by centrifugation, suspensions were dried on glass slides. For immunohistochemical procedures in the control group a mixture of three different candida isolates suspended in PBS and dried on glass slides was used.

For immunohistochemistry smears of native vaginal secretions were covered by $3 \%$ hydrogen peroxide for 5 minutes to block endogenous peroxidase activity. After PBS rinsing and 10 minutes preincubation with Porcine Serum (GIBCO, UK) specimens were incubated for 1 hour with rabbit anti human IgA antibody diluted 1:100 (Dako; Code No A 262 Glostrup, Denmark). The buffer was changed and the 1:50 diluted swine anti rabbit antiserum (Dako, Z 196, Glostrup, Denmark) was added and incubated for 30 minutes. Sections were then washed three times in PBS and incubated with PAP complex (Dako PAP rabbit Z 113) diluted 1:150 for 30 minutes. All antibodies were of the IgG class. For staining we used a staining kit containing 3-amino 9-ethyl carbazole (Tissue Gnost AEC Chromogen 20017, Merck). Sections were counterstained with haemalaun.

Suspensions of subcultured yeasts were subjected to immunohistochemistry as described above after incubation with the reconstituted vaginal secretion sample of the respective patient for 30 minutes. In the control group a mixture of three different Candida species strains was incubated with the samples. Judgement of immunohistochemical preparations was carried out by an investigator blinded to the clinical diagnosis. Staining intensity of vaginal smears and subcultures was assesed on a three point scale $(+,++$, $+++)$ with negative samples marked with a minus. In addition, the percentage of positively staining blastospores in subcultures was also documented. Controls included dilution series and incubation of the subcultures with purified fractions of IgA instead of vaginal secretion as well as staining of chronic inflammatory gastric mucosa. As negative controls the primary antibody was omitted and smears of the subcultures were subjected to the immunohistochemical procedure without preincubation with vaginal secretion. In addition the primary antibody was substituted with an inappropriate antibody, i.e. S100 antibody (diluted 1:100).

The Wilcoxon 2-Sample Test (Normal Approximation) was used for Significance analysis. A result for which $p$ was $>0,05$ was regarded as non significant. For correlation analysis Spearman Correlation Coefficients were evaluated. A positive or negative correlation was assumed when $r$ was $>0.7$ or $<-0 \cdot 7$.

\section{Results}

VVC was caused by Candida albicans (Ca) in 27 patients and by Candida glabrata $(\mathrm{Cg})$ in 2 cases. In one patient $\mathrm{Ca}$ and $\mathrm{Cg}$ could be isolated simultaneously. $\mathrm{Cg}$ was exclusively found in group 1.

IgA was detected in $45(75 \%)$ of 60 samples of cervicovaginal secretions. Median levels were 2.5 (range $0-15.8$ ) $\mathrm{mg} / \mathrm{dl}$ and 3 (range $0-8$ ) $\mathrm{mg} / \mathrm{dl}$ for group 1 and group 2 respectively. Nine negative samples were found in group 1 and six in group 2 . Negative results were confirmed by a second testing. Serum IgA levels, available in 59 women, revealed median levels of $200 \mathrm{mg} / \mathrm{dl}$ (range $85-496 \mathrm{mg} / \mathrm{dl}$ ) in group 1 and $210 \mathrm{mg} / \mathrm{dl}$ 
Figure 1 Immunostaining of smear preparations from the vaginal mucosa, intense Ig $A$ coating of hyphae (a) and blastospores $(\Rightarrow)(b) ; P A P$ method. Haematoxylin. magnification $\times 1000$.

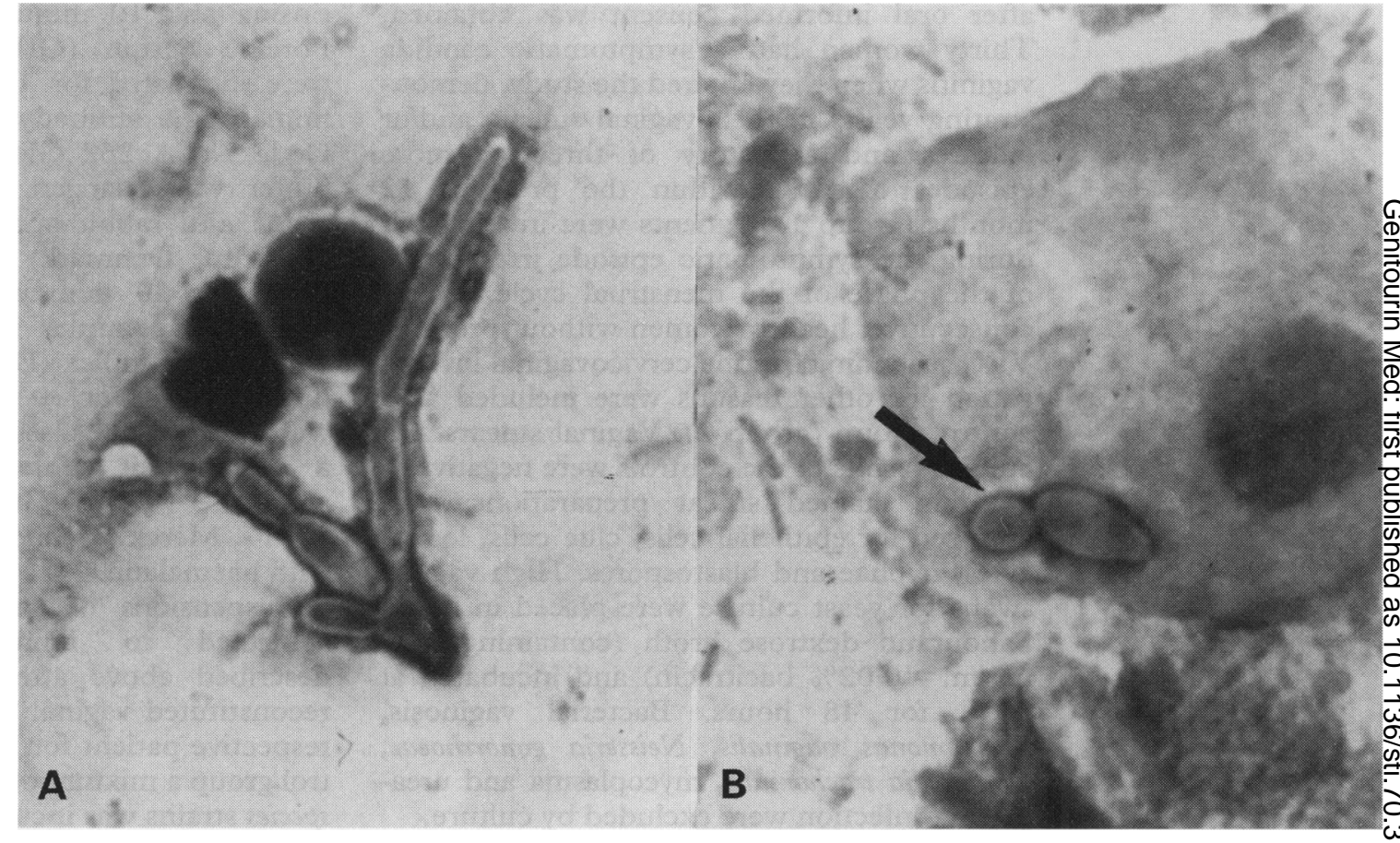

(range $42-543 \mathrm{mg} / \mathrm{dl}$ ) in group 2 respectively. No significant difference in the IgA levels of cervicovaginal secretions $(p=0.2381)$ or serum ( $p=0.5796$ ) was found between the two diagnostic groups (table 1). The IgA levels between serum and secretions showed no positive correlation $(\mathrm{N}=59 ; \mathrm{r}=$ -0.00012 ). Immunohistochemistry of vaginal smears revealed blastopores and/or hyphal elements in vaginal smears of 19 patients of group $1(63 \%)$. The remaining 11 patients of group 1 and the entire control group were negative on direct microscopy. Most of the visualised morphotypes of candida revealed IgA coating on their surfaces (fig $1 \mathrm{a}, \mathrm{b}$ ). Bacteria, in particular lactobacilli, stained variably as well as cervicovaginal mucus. Epithelial cells were unreactive. For detailed data of staining intensity see table 2 .

Due to $\mathrm{Ca} / \mathrm{Cg}$ coinfection in one patient 61 subcultures of 60 patients were investigated immunohistochemically. Fifty eight revealed IgA loaden blastospores after incubation with patients' vaginal secretion (table 2). Three

Table 1 Median IgA levels in serum and cervicovaginal secretion according to diagnostic group

\begin{tabular}{|c|c|c|c|c|c|c|}
\hline \multirow[b]{2}{*}{ Group } & \multicolumn{3}{|c|}{ IgA-Serumt } & \multicolumn{3}{|l|}{ IgA Secretiont } \\
\hline & $N$ & Median & Range & $N$ & Median & Range \\
\hline $\begin{array}{l}1 \\
2\end{array}$ & $\begin{array}{l}30 \\
29\end{array}$ & $\begin{array}{l}200 \\
210 \\
p^{\star}=0.5796\end{array}$ & $\begin{array}{l}85-496 \\
42-543\end{array}$ & $\begin{array}{l}30 \\
30 \\
p^{\star}=0.2381\end{array}$ & $\begin{array}{l}2 \cdot 5 \\
3\end{array}$ & $\begin{array}{l}0-15 \cdot 8 \\
0-8\end{array}$ \\
\hline
\end{tabular}

†Data given in $\mathrm{mg} / \mathrm{dl}$

*Calculated by Wilcoxon 2- Sample Test (Normal Approximation)

Table 2 Results of immunohistochemistry of vaginal smears and subcultures, focusing on staining intensity as well as percentage of blastospores labelling for IgA

\begin{tabular}{|c|c|c|c|c|c|c|c|c|c|}
\hline \multirow[b]{2}{*}{ Patients } & \multirow[b]{2}{*}{ Specimen } & \multirow[b]{2}{*}{ neg } & \multicolumn{3}{|c|}{ Intensity } & \multicolumn{4}{|c|}{ Percentage } \\
\hline & & & + & ++ & +++ & neg & $<50$ & $50-80$ & $>80$ \\
\hline $\begin{array}{l}\text { group } 1 \\
\text { group } 2\end{array}$ & $\begin{array}{l}\text { vag. smear } \\
\text { subculture } \\
\text { subculture }\end{array}$ & $\begin{array}{l}0 \\
3 \\
0\end{array}$ & $\begin{array}{r}4 \\
14 \\
12\end{array}$ & $\begin{array}{r}10 \\
7 \\
15\end{array}$ & $\begin{array}{l}5 \\
7 \\
3\end{array}$ & $\begin{array}{l}3 \\
0\end{array}$ & $\begin{array}{l}5 \\
3\end{array}$ & $\begin{array}{l}10 \\
10\end{array}$ & $\begin{array}{l}13 \\
17\end{array}$ \\
\hline
\end{tabular}

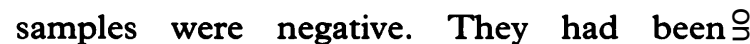
obtained from two patients suffering from $\mathrm{Ca}-$ and $\mathrm{Cg}$ infection respectively. One patient $\subsetneq$ who sustained $\mathrm{Ca} / \mathrm{Cg}$ coinfection revealed ${ }^{\overparen{ }}$ negative staining for her $\mathrm{Cg}$ subculture $\vec{\emptyset}$ whereas her $\mathrm{Ca}$ subculture was weekly? positive. A striking inter- and intra-strain variability of blastospore size and configuration was noted. Even in subcultures with $80-100 \% \overline{0}$ positivity isolated rather big cells stained negative (fig 2). Comparing subcultures with vagi- $\varrho$ nal smears, staining intensity was invariably $\overrightarrow{\overrightarrow{0}}$ weaker in the subcultures.

Immunoglobulin precipitates were mainly found on the cell surface. Reproducibility of these observations was provided by repeated testing. No correlation between staining intensity of blastospores in subcultures and IgA content of vaginal secretion was found $(N=60 ; r=-0.01578)$. In regard to the variables group, IgA in serum, IgA in cervicovaginal secretion and immunhistochemistry of subcultures a trend analysis showed no correlation between the 4 parameters (Kendall ${ }^{\circ}$ Tau Correlation Analysis). Staining intensity ${ }^{N}$ correlated with concentration of antisera. $\mathbb{W}$ Omission of the primary antibody, incubation

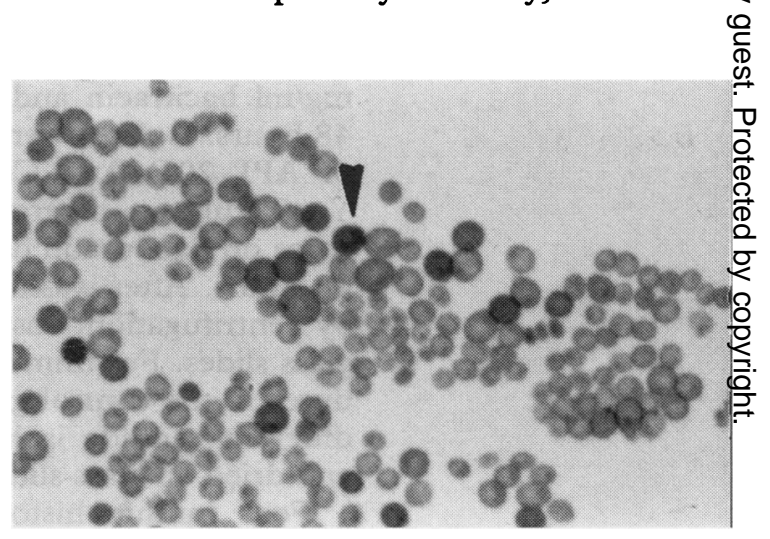

Figure 2 Subcultured yeasts; note intrastrain variability of blastospore size, large unreactive blastospores ( ) scattered among intensely labelled blastospores. PAP method. Haematoxylin; magnification $\times 300$. 
with the inappropriate antibody as well as omission of preincubation with vaginal secretion gave negative results. Preincubation of subcultures with purified IgA produced clearly positive staining of blastospores.

\section{Discussion}

Although no statistically significant differences in sIgA levels of cervicovaginal secretions and sIgA coating of candida cells could be demonstrated between women with recurrent symptomatic VVC and healthy controls, yet several interesting observations confirmed the importance of host parasite relationship in aetiopathogenesis of recurrent VVC.

From the immunofluorescence studies of oral bacteria performed by Brandtzaeg et $a l_{9}{ }^{11}$ and the light- and electronmicroscopic investigations by Metze et al ${ }^{12}$ we know that microbes of skin as well as mucous membranes bind immunoglobulins on their surfaces. We confirmed these findings in all 19 patients $(63 \%)$ in whom yeast or hyphal forms of $\mathrm{Ca}$ had been detected in vaginal smears. In spite of the superior value of direct microscopy, several studies have consistently demonstrated that up to $50 \%$ of patients with culture positive, symptomatic candidal vaginitis will have negative microscopy. ${ }^{13}$ These data prompted us to study in addition to fresh isolates subcultured organisms.

Variations in blastospore size and shape, a basic finding in all subcultures, can be interpreted as quantitative and qualitative variations in apical and general cell wall synthesis. ${ }^{14}$ It can be assumed further that within a given strain variations in blastospore size represent different ages, with the bigger cells being the older ones. A negative correlation was noted between the size of the blastospore and the staining intensity, which might reflect a loss of immunoglobulin receptors in the ageing yeast and would explain why even in cases with $80-100 \%$ positivity large IgA negative blastospores were found. In addition staining of subcultures was invariably weaker than of vaginal smears possibly due to a receptor loss under in vitro conditions. Immunohistochemistry of subcultures was negative in three patients of group 1 , suffering from infection with $\mathrm{Ca}, \mathrm{Cg}$ and coinfection of both species respectively. Vaginal smears were negative for fungal elements in two cases, which prohibited assessment of staining conditions under in vivo conditions. Vaginal smear of the patient suffering from $\mathrm{Ca} / \mathrm{Cg}$ coinfection revealed hyphae weakly positive for IgA as well as completely negative yeasts. In accordance subculture of $\mathrm{Ca}$ showed weak IgA binding of blastospores, whereas subculture of $\mathrm{Cg}$ was completely negative. These findings are remarkable because they might indicate that strain specific binding defects play a role in recurrent vaginitis.

The fact that IgA labelling of blastospores was shown in 58 out of 61 candida subcultures was inconsistent with the results of single radial immunodiffusion indicating measurable IgA levels in only $75 \%$ of cervico- vaginal secretions. This discrepancy can be interpreted as insufficient sensitivity of single RID in quantitative analysis of IgA in heterogenous secretions like cervicovaginal mucus.

In accordance with the results of Gough et al. ${ }^{15}$ we found no statistically significant difference in the serum $\operatorname{IgA}$ and $\operatorname{IgA}$ in vaginal secretions between the two groups. Yet there is no consensus about the implications of vaginal infection on local SIgA production. Chipperfield et $a l^{16}{ }^{17}$ have shown that genital infection leads to increased numbers of immunoglobulin secreting plasma cells and increased IgA and IgG levels in cervical mucus. Mathur et al ${ }^{18}$ report high sIgA serum levels in patients with VVC most likely due to passive diffusion from vaginal mucosa into systemic circulation. Waldmann et al. ${ }^{19}$ describe lowering of antibody titres in five women whose secretions had been culture positive for $\mathrm{Ca}$. Although it is not stated whether these women were symptomatic or not these results could be explained by a consumption phenomenon.

$\mathrm{Ca}$ forms part of the resident flora of the human digestive tract, stimulating continous production of specific IgA. Therefore specific IgA can be detected in cervicovaginal secretions of healthy women who never had vaginal candidiasis.

Adherence, proteinase production and phenotypic switching are the most discussed candida virulence factors. All $\mathrm{Ca}$ strains adhere equally well to exfoliated vaginal cells, but there is a considerable individual variation in vaginal cell receptivity to candida in adherence assays. Nevertheless, vaginal cells from women with recurrent VVC do not show increased cell avidity or affinity kinetics for candida. $^{20}$ However, germination has been demonstrated to influence candidal virulence substantially. ${ }^{4}$ Since the original description of switching colonial morphologies by Slutsky et al, Soll et al observed additional systems of in vitro reversible switching of colonies, where the new cells had acquired superior virulence in terms of an increased capacity to adhere, germinate and produce proteases. ${ }^{21} 22$ In vivo these results could only partly be confirmed. ${ }^{23}$

It is well known that $\mathrm{Ca}$ and some other pathogenic candida species secrete acid proteinase which degrades various host proteins and therefore is potentially involved in the pathogenicity of the organism. ${ }^{24}$ It has been shown by Cassone et al that proteinase secretion is particularly relevant in the pathogenesis of VVC. ${ }^{25}$ The authors found that $\mathrm{Ca}$ isolates from patients with active vaginitis were significantly more proteolytic than from carriers. Immunoglobulins are most easily cleaved in the "hinge" region which is located between the first and second constant region domains. The fragments produced comprise either two univalent $\mathrm{Fab}$ or one divalent $F(a b) 2$ fragment and one $F c$ fragment. Whereas antigen-binding activity is associated with the Fab fragments biological activities are associated with the $\mathrm{Fc}$ fragment. Also sIgA is cleaved easily by candida proteases. ${ }^{26}$ In our 
immunohistological investigations we used an antibody specific for alpha-chains. Labelling for IgA included biologically active, intact IgA molecules as well as immunologically useless IgA fragments whenever present along the yeasts' surface. Therefore it is impossible to draw quantitative conclusions from staining intensity of immunohistochemistry on the biological activity of the local immunsystem. To elucidate further mucosal immunoglobulin-mediated immunity in recurrent vaginal candidiasis differentiation and quantitation of intact IgA molecules from IgA fragments in cervicovaginal secretions should be the subject of further investigations.

In conclusion recurrent VVC cannot be attributed to IgA deficiency. In some cases an IgA coating defect of yeasts might be involved. In addition inactivation of the $\operatorname{IgA}$ molecule by candida proteases might be of pathogenetic importance.

The authors thank F. Pieczkowski for her technical assistance and I. Agstner and M. Mittelböck for statistical expertise.

1 Odds FC. Pathogenesis of Candidosis. In: Odds FC, ed Candida and Candidosis 2nd ed. London, Bailliere, Tindall. 1988;269-78.

2 Odds FC. Candidosis of the Genitalia. In Candida and Candidosis 2nd ed. London, Bailliere, Tindall. 1988; 124-35.

3 Sobel JD. Pathophysiology of Vulvovaginal Candidiasis. $千$ Reprod Med 1989;34,Suppl:572-80

White DJ, Emens M, Shahmanesh M. Recurrent vulvovaginal candidosis. Int $\mathcal{F}$ STD AIDS 1991;2:235-9.

5 Milne JD, Warnock DW. Effect of simultaneous oral and vaginal treatment on the rate of cure and relapse in vaginal candidosisans. $B \mathcal{f}$ Venereal Dis 1979;55:362-5.

6 Witkin SS, Yu RI, Ledger WJ. Inhibition of Candida albicans-induced lymphocyte proliferation by lymphocytes cans-induced lymphocyte pro lifation by lymphocytes abstet Gynecol 1983;147:809-12.

7 Witkin SS, Hirsch J, Ledger WJ. A macrophage defect in women with recurrent candida vaginitis and its reversal in vitro by prostaglandin inhibitors. Am $\mathcal{F}$ Obstet Gynecol
1986;155:790-5.

8 Tomasi TB. The Secretory Immune System. In Stites DP, Stobo JD, Fudenberg HH, Wells JV, eds: Basic \& Clinical Immunology Lange Medical Publication, Los Altos. 1982;198-208.

9 Rebello R, Green FHY, Fox H. A study of the secretory immune system of the female genital tract. $\mathrm{Br} \mathcal{F}$ Obst Gynaecol 1975;82:812-6.

$10 \mathrm{McNabb}$ PC, Tomasi TB. Host defense mechanisms at mucosal surfaces. Ann Rev Microbiol 1981;35:477-96.

11 Brandtzaeg P, Fjellanger I, Gjeruldsen ST. Adsorption of immunoglobulin A onto oral bacteria in vivo. $\mathcal{F}$ Bacteriol 1968;96:242-9.

12 Metze D, Kersten A, Jurecka W, et al. Immunoglobulins coat microorganisms of skin surface: a comparative immunohistochemical and ultrastructural study of cutaneous and oral microbial symbionts. $\mathcal{F}$ Invest Dermatol 1991;96:439-45.

13 Bertholf ME, Stafford MJ. Colonization of Candida albicans in vagina, rectum and mouth. If Family Pract cans in vagina,

14 Staebell $M$, Soll DR. Temporal and spatial differences in cell wall expansion during bud and mycelial formation in

15 Gough PM, Warnock DW, Richardson MD, et al. IgA and IgG antibodies to Candida albicans in the genital tract secretions of women with or without vaginal candidosis. Sabouraudia 1984;22:265-71.

16 Chipperfield EJ, Evans BA. The influence of local infection on immunoglobulin formation in the human endocervix. Clin Exp Immunol 1972;11:219-23.

17 Chipperfield EJ, Evans BA. Effect of local infection and oral contraception on immunoglobulin levels in cervical mucus. Infect Immun 1975;11:215-21.

18 Mathur S, Virella G, Koistinen J, et al. Humoral immunity in vaginal candidiasis. Inf $\mathcal{E}$ Immun 1977;15:287-94.

19 Waldmann RH, Cruz JM, Rowe DS. Immunoglobulin levels and antibody to Candida albicans in human cervicovaginal secretions. Clin Exp Immunol 1971;9:427-34.

20 Calderone RA. Host parasite relationship in candidosis. Mycoses 1989;32:(Supp12),12-17.

21 Slutsky B, Buffo J, Soll DR. High frequency switching of colony morphology in Candida albicans. Science 1985;230:666-9.

22 Soll DR. High frequency switching in Candida albicans and its relations to vaginal candidiasis. Am $\mathcal{F}$ Obstet Gynecol 1988;158:997-1001

23 Soll DR, Galeist R, Isley S, et al. Switching of Candida albicans during successive episodes of recurrent vaginitis. f Clin Microb 1989;27:681-90.

24 Rüchel $R$. A variety of candida proteinases and their possible targets of proteolytic attack in the host. Zentralb Bakteriol Mikrobiol Hyg $(A)$ 1984:257:266-74.

25 Cassone A, De Bernardis F, Mondello F, et al. Evidence for a correlation between proteinase secretion and vulvovaginal candidosis. $\mathcal{f}$ Infect $D$ is 1987;156:777-83.

26 Rüchel $R$. Cleavage of immunoglobulins by pathogenic yeasts of the genus candida. Microbial Sci 1986;3:316-9. 